\title{
المداخل السيكولوجية وتطبيقاتها في تدريس اللغة العربية
}

\author{
Moh. Anwar \\ mohanwar82@gmail.com \\ Dosen STIT Aqidah Usymuni Terate Sumenep
}

\begin{abstract}
Until now, there are still many Arabic teachers who do not have an adequate understanding of the psychological approach to learning and how to apply it in learning Arabic. This condition causes the learning method applied is still traditional and conventional. As a result, many students feel bored and find it difficult to digest the subject matter so that learning outcomes are not optimal. By understanding the psychological approach in learning Arabic, teachers are expected to be able to understand the nature of the subject matter and choose and determine the appropriate method according to the psychological tendencies of students in relation to the subject matter given.

Through this paper, the author tries to refresh the memory and understanding of Arabic teachers about various psychological approaches and their application efforts in learning Arabic. There are at least four kinds of learning psychology approaches proposed, namely the behaviorism approach, the cognitivism approach, the humanism approach and the constructivism approach. The four kinds of approaches are comprehensively reviewed and their application in Arabic learning.
\end{abstract}

Keywords: Approach, Psychology, Behaviorism, Cognitivism, Humanism, Constructivism

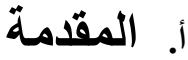

توضح الواقعة الحالية أنه حتى اليوم لا يزال هناك العديد من المعلمين الذين

يطبقون الطرق التقليدية والقديمة في التدريس بدون النظر والاعتماد على المفاهيم الكافية

للنظريات الفلسفية عن المواد التي يتم تدريسها. ونعني بالنظريات الفلسفية هي المعارف

التي ترتبط بطبيعة المواد التعليمية الذي يُنظر إليها من وجهات نظر مختلفة، وهو ما يُعرف عندنا بالمدخل. يمكن بالتأكيد تناول موضوع ما ومر اجعته من وجهات نظريات مختلفة، ومن خلال وجهة النظر هذه، يمكن للمدرس اختيار وتحديد أساليب وتقنيات واستر اتيجيات التعليم التي تعتبر أكثر ملاءمة وفعالية للتطبيق. على سبيل المثال، دروس اللغة العربية التي تُرى من وجهة النظر أو المدخل التواصلي، ووفقًا لهذا المدخل، فإن اللغة ليست سوى أداة 
للتواصل تستخدم كوسائل لنقل الرسائل والأغر اض بين الأشخاص الذين يستخدمون تلك اللغة. و اعتمادا بهذا المدخل أن تعليم اللغة موجه بشكل أكبر إلى قدرة الطلاب على التواصل ماللغة التي يتم تدريسها باستخدام الأساليب و الطريقة المناسبة مثل الطريقة المباثرة ( Direct Methode). و على عكس ذللك، فإن المدخل البنيوية، يعتقد أن اللغة تتكون من عناصر أو مكونات، تعليم اللغة هو في الأساس تعليم إتقان هذه العناصر. من خلال إتقان الطلاب عناصر اللغة، يمكن لهم استخدام اللغة بشكل صحيح. وتطبيق هذا المدخل في التعليم هو أن الأولويات التي يجب تدريسها هي إتقان البنية والتراكيب النحوية للغة، و القواعد الصوتية، و الصرفية، والنحوية، والدلالية. علاوة على ذلك، فإن اختيار الطريقة التي تتوافق مع المدخل البنيوي هي طريقة الترجمة النحوية (القو اعد و الترجمة)، و هي طريقة نؤكد على فهم الطلاب نحو وظائف كل من الجمل أو الكلمات من حيث جانبها النحوي (القو اعد) ثم يستمر بترجمة تلك الجمل إلى لغة الطلاب. وبجانب افتقار سيطرة المعلمين حول المدخل، كذلك، لا يمكنه في كثير من الأحيان على التمييز بين العديد من الأساليب الحالية بل ويميلون إلى التلبيس. ربما ينبع هذا أيضًا من بعض الكتب التي أصبحت كالمر اجع، و غالبًا ما تُظهر بعض الكتب أن المدخل في تعليم اللغة العربية غالبًا ما يستوعب عدة أنواع من المداخل في موضوع فرعي واحد من "المدخل"، على الرغم من أن هذه الأنواع لها موضوعات فرعية خاصة بها. على سبيل المثال، المدخل الإنساني، و المدخل البنائي، و المدخل التواصلي، و المدخل التقني و المدخل السلوكي، وغالبًا ما يُنظر إلى كل منها على أنه موضوع فرعي، أي مداخل التعليم. ومع ذللك، إذا قمنا بتتبعه، فلكل نوع من المدخل فهناك تصنيف رئيسي ووجهة نظر مختلفة. يتم تضمين المداخل السلوكية والبنيوية و الإنسانية والمعرفية في مجموعة "المدخل النفسي". وفي الوقت نفسه، يتم تضمين المدخل البنيوية والمدخل التواصلية في مجموعة "المدخل 
إستنادا على ما ذكر، ستحاول هذه الأطروحة على استكثاف ثلاث قضايا رئيسية، و هي؛ أو لاً، بشرح مفاهيم مدخل التعليم. ثانيًا، قمنا بتحليل كل أنو اع الدذاخل في تعليم اللغة التي تثمل الدداخل النفسية والدداخل اللغوية. ثالثا : تطبيق كل من كلا المدخلين في تعليم

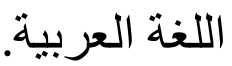

\section{ب. المدخل في تعليم اللغة العربية}

تأتي كلمة مدخل من اللغة الإنجليزية، وهو approach الذي في اللغة بمعنى "المدخل" أو

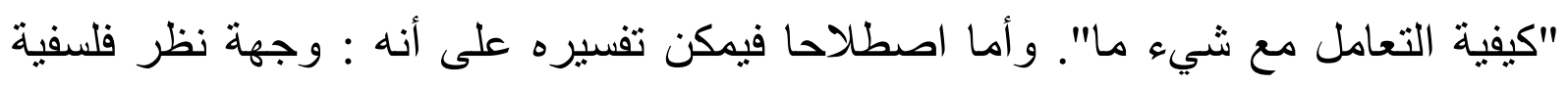

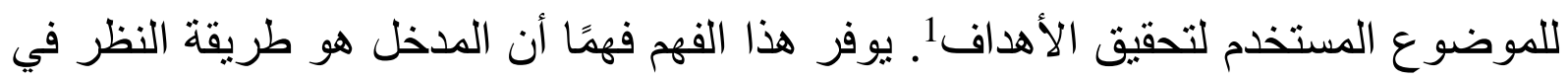
موضوع ما. على سبيل المثال، أدى منظور الثخص لعملية التعلم إلى مصطلح مدخل التعلم ، حيث تكون لكل شخص بالتأكيد وجهة نظره الخاصة في رؤية مادة مختلفة عن وجهة الفئا النظر التي يستخدها الأثخاص الآخرون، اعتمادًا على الجانب الذي يرونه فيه. بمعنى آخر ، أن المدخل فيما يتعلق بالتعلم هو نقطة البداية أو وجهة نظر الثخص تجاه عملية التعلم

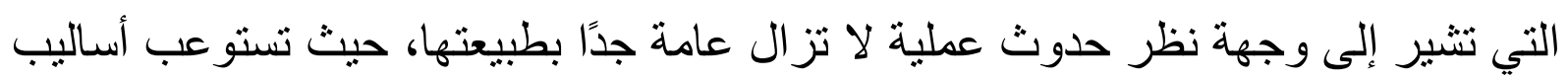
التعلم مع نطاق مدخل محدد وتلأمها وتقويها وتؤسسها. وفي الوقت نفسه، أوضح Suyono و Hariyanto فكرة عن نعريف مدخل التعلم، بأنه

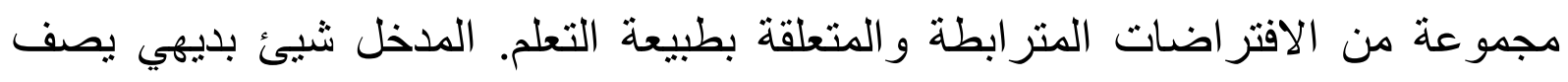
المعايير و الخصائص للمادة التي يتم تدريسها2. من خلال تعريف مدخل التعلم لمثل هذا، تبين لنا أنه بشمل على خلفية نفسية وخلفية تربوية تصبح مرجعا عقلانيا واعتبار ا في اختبار طرق التعليم التي سيتم استخدامها وتطبيقها من قبل المعلمين نحو الطلاب. تم تطوير الددخل من قبل خبر اء التربويين من خلال دراسات

\footnotetext{
${ }^{1}$ Novan, Ardy Wiyani.. Desain Pembelajaran Pendidikan: Tata Rancang Pembelajaran Menuju Pencapaian Kompetensi, (Yogyakarta: Ar-Ruzz Media, 2013), Hlm. 35

${ }^{2}$ Ahmad Fuad Efendy, Metodologi Pengajaran Bahasa. (Malang : Misykat, 2012), Hlm. 8
} 
نفسية وتربوية في فترة زمنية طويلة لإنتاج المفاهيم عن المدخل الموثوق ويمكن مسؤوليته أكاديميًا و علميًا. علاوة على ذللك، فإن الأساليب التي تم تصور ها بعناية يتم محاولة موازنتها مع الحقائق الفعلية للتعليم من خلال التعاون مع ممارسي التعليم والمهتمين للحصول على الإتفاق بينهم حول كيفية التعليم.

بشكل خاص فيما يتعلق بتعليم اللغة، يعرّف إدوارد أنتوني (1963)، كما نقله أحمد فؤاد أفندي المدخل على النحو التالي: مجموعة من الافتراضات المتعلقة بطبيعة اللغة وتعليمها وتعلمها3. تظهر الافتر اضات أو المعتقدات بهذا التعريف نشير على أثكالها المختلفة وفقًا لوجهات نظر كل منها، من بين أمور أخرى ؛ الإفتر اض الذي يعتقد أن اللغة هي عادة مثل العادات الأخرى، أو الافتر اض الذي يرى اللغة كوسيلة للتواصل، أو الافتر اض الذي يرى أن اللغة تتكون من النظام أو القو اعد التي يجب طاعتها. يعرّف Tarigan بشكل أكثر عملية أن المدخل باعتباره افتراضًا بديهيًا حول طبيعة اللغة وتعليمها وتعلمها و الذي يستخدم كأساس في تصميم وتنفيذ عملية التعليم و التعلم. فبالإعتماد على المدخل، يتم تصميم وتتفيذ جميع الإجر اءات و الأساليب و الاستر اتيجيات وخطوات التعلم. هذا هو المكان الذي يحتاج إلى شرح أكثر وضوحًا وقابلية للنطبيق حول طبيعة مدخل تعليم اللغة وجهود تطبيقها في تعليم اللغة العربية. على وجه التحديد، هناك طريقتان لتعليم اللغة العربية يستخدمها العديد من ممارسي ونُشَطاء تعليم اللغة العربية في الأعمال المكتوبة (الكتاب) وفي مستو اهم العملي في أنشطة التعليم والتعلم. وكلا المدخلين هما المدخل النفسي والمدخل اللغوي. يشرح المدخل النفسي عملية التعليم لدى الثخص من منظور علم النفس، ويسعى المدخل اللغوي إلى شرح طبيعة تعليم اللغة من وجهة نظر علم اللغة، كما سيتم وصفهما في ما 


\section{ج. المدخل السيكولوجي في تعليم اللغة العربية}

يمكن تعريف المدخل السيكولوجي للتعلم على أنه : الافتر اضات التي يعتقدها بعض علماء علم النفس المنز ابط فيما يتعلق بطبيعة التعلم والتعليم في الثخص4. المدخل هو خلفية فلسفية حول الموضوع الذي سيتم تدريسه. في عملية التعلم لا يقتصر الأمر على إتقان الحقائق أو المفاهيم في مجال المعرفة فحسب، بل يشمل أيضًا المشاعر المتعلقة بالعواطف والمودة والكر اهية و الرغبة و الإعجاب و الميول و الروحانية. ففي سيكولوجية التعليم، الذي يصبح تركيز ا عليه هو لماذا، ومتى، وكيف تتم عملية التعلم في الكائن الحي. كل الكائنات الحية لها قدراتها الخاصة وطرق تعلمها بناءً على ميو لها النفسية. بناءً على هذا الوصف، إذا كان ما تريد مناقتشه هو المدخل النفسي، فالذي يجب شرحه هو الافتراضات النفسية. لأن كلا من علم النفس لديه معتقدات معينة حول التعلم. فتدفق السلوكية له افتر اضاته المختلفة دن تدفق المعرفية و البنائية. لكل معتقد عو اقب مختلفة للتعلم، بدءًا من اختبار المو ادو الأساليب و الاستر اتيجيات و التنفيذ و المتابعة.

تعليم اللغة هو عملية إتقان اللغة، سواء كان في اللغة الأولى أو في اللغة الثانية. يمكن أن تحدث عملية اكتساب اللغة بشكل طبيعي (اكتساب اللغة) أو تُعرف أيضًا بالاكتساب، أو رسميًا و مخططًا (تعليم اللغة) وهو ما يُعرف بالتعليم. يحدث الاكتساب بشكل طبيعي عندما يكتسب الطفل لغته الأولى والتي تحدث بشكل طبيعي بدون خطة وبدون وعي. بينما يحدث التعليم عندما يتعلم الثخص لغة ثانية من خلال عملية مخططة ومصممة بهذه الطريقة في عملية التعليم و التعلم بشكل رسمي وغير رسمي. لذلك، من أجل الحصول على فهم شامل للعمليات النفسية و افتر اضات المدخل التي تؤمن بها بعض الفهم النفسي، سوف نراجع بعض المذاهب في علم النفس التي لديها الكثير من الألوان في تعلم اللغة وجهود تطبيقها في تعلم اللغة العربية.

\footnotetext{
${ }^{4}$ Pranowo, Teori Belajar Bahasa, (Yogyakarta : Pustaka Pelajar, 2014) hlm. 26
} 
1. المخل السلوكي

المؤسس لهذه الدرسة هو العالم الروسي إيفان بتروفيتش بافلوب (1849-1936) المشهور بتجاربه التي ربطت المنبه الأساسي (الغذاء) والمحفزات الثانوية (الأضواء والأجراس) باستجابة (لعاب) الكلاب التي استخدمت كحيوانات تجريبية. بناءً على بحث التهات

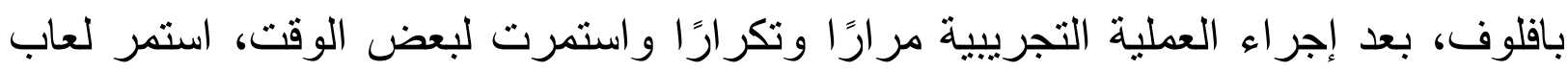
الكلب في النهاية في التدفق عندما كان الضوء مضاءًا ولو كان بدون طعام. الاستتناج الذي توصل إليه بافلوف بناءً على هذه التجارب هو أن الكلاب قادرة على على التعلم من العادات. مع الأضواء المتكررة أو أصوات الجرس، هو مثير ينتج في النهاية استجابة

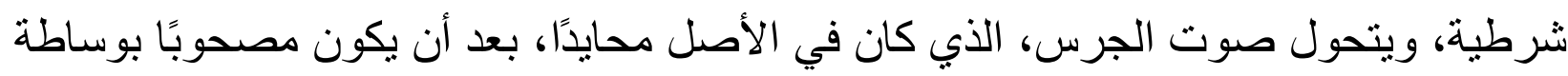
على شكل مسحوق اللحم، تدريجياً إلى قوة قادرة على توليد استجابة. وفقًا لبافلوب، يمكن أيضًا تطبيق النتائج التجريبية على الإنسان في عملية التنديس و التعلم. مدخل التعلم السلوكي هو الدذخل الذبي يركز اهتمامه على السلوك البشري. نظرت النّات

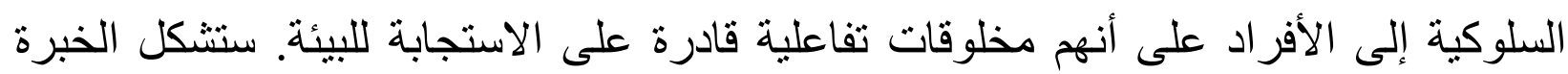

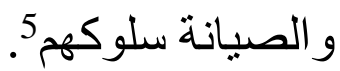

فمن سمات مدخل التعلم السلوكي تفضيل الأولوية للعناصر والأجزاء الميكانيكية الصغيرة. يؤكد هذا الدذخل على دور البيئة ويؤكد على تكوين ردود الفعل أو الاستجابات. بالإضافة إلى ذلك، فإنه يؤكد أيضًا على أهمية الممارسة ونتائج التعلم الميكانيكي، ويؤكد على الثى دور القدرة نتيجة التعلم التي تم الحصول عليها هي ظهور السلوك المطلوب6. وفي هذا المذل غالبا ما تسمى بالمثير و الإستجابة، كما سنشرح كلا منهما فيما بعد :

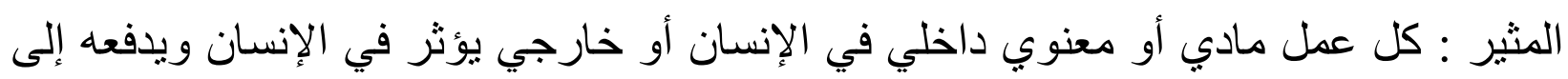
التصرف بشكل من الأشكال، وهو عند هاريمان بأنه أب صورة للطاقة تنتج استجابة، أو اي

${ }^{5}$ Abdul Wahab Rosyidi \& Umi Mahmudah, Aktive Lerning Dalam Pembelajaran Bahasa Arab, (Malang : UINMALANG PRESS, 2008), hlm. 38

${ }^{6}$ Ibid, 39 
طاقة خارجية بالنسبة لعضو الإستقبال تؤثر عليه وتستثيره. ويعرفه سكينر على أنه نوع من الأحداث البيئية التي لا يمكن تحديدها منفصلة عن ملاحظات أسلوب معين من أساليب الكائن

أما الإستجابة : فهي ردة الفعل الناتج عن المثير كفعل طبيعي يتصف به الكائن الحي، أي طريقة التفاعل الإيجابي مع المثير الحاصل عند الإنسان، وتعرف بيولوجيا بأنها : تقلص عضلي أو إفراز غدي (من الغدد) أو أي نشاط آخر ينتج عنه استثارة.8. على سبيل الإختصار ، يمكن وصف مدخل التعليم السلوكي على النحو التالي ؛ أ) التعلم هو تغيير في السلوك ب) يجب أن يكون السلوك ملحوظًا. ج) يهتم جانب المدخلات في شكل المثير و المخرجات في شكل استجابة. د) تتمثل وظيفة العقل في إنشاء هياكل معرفية قائمة من خلال عملية تفكير يمكن تحليلها وفرزها. ه) العادة و الممارسة ضرورية في التعلم. و) ما يحدث بين المثير والاستجابة يعتبر غير مهم لأنه لا يمكن ملاحظته. ز) كل ما يمكن ملاحظته هو المثير والاستجابة. ح) تعد الفشل أو عدم القدرة على زيادة المعرفة على أنه فنشل تجب معاقبته. ط) يتطلب تطبيق هذا المدخل من الطلاب إعادة التعبير عن المعرفة التي تم تعلمها في شكل تقارير أو اختبار ات. يتبع عرض الموضوع ترتيب الأجزاء ككل. يؤكد التعلم والتقييم على هـ

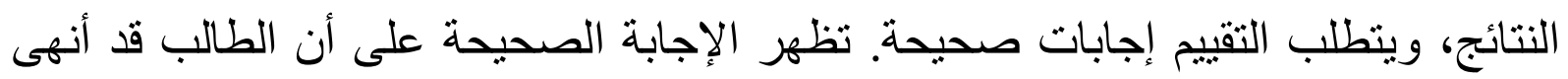
الدر اسة.9. ي) تعتمد عملية التعلم بشكل كبير على العو امل الخارجية منه، لذلك يحتاج إلى المثير من المعلم. 7 عبد المجيد عيساني، نظريات التعلم وتطبيقها في علوم اللغة (القاهرة : دار الكتب الحديث، 2012) ص. 67 9 , Psikologi Pembelajaran Bahasa Arab (Pekanbaru: Almujtahadah Press, 2010), 126 - 127 
ك) يتم تحديد نتائج التعلم إلى حد كبير من خلال عملية التقليد والتكرار و التعزيز. ل) يجب أن يمر التعلم بمراحل معينة، شييًا فثينًا، المراحل السهلة تسبق المراحل الأكثر

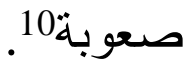

أما بالنسبة لتطبيق المدخل السلوكي في التعلم، حيث يرى خبر اء علم النفس أن تعلم اللغة يتم

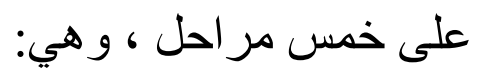

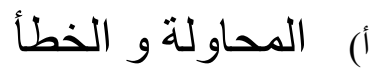
ب) التذكر و المحافظة

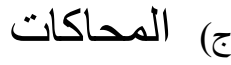

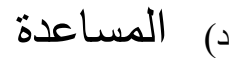

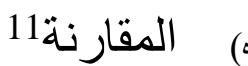

من الخطو ات الخمس ، يمكن الإستنتاج بأن تعليم اللغة في الأساس هي عملية تكوين العادات. ففي المدخل السلوكي، يصبح كل السلوك البشري سلوكًا لغويًا يصبح مظهرًا من مظاهر المثير و الاستجابة التي تتم بشكل مستمر في العادة. بناءً على هذا المدخل، يتم تنفيذ تعليم اللغة من خلال إعطاء الأولوية لإدخال مهار ات الاستماع و التحدث على المهار ات الأخرى، وتوفير التدريبات و استخدام اللغة بشكل نشط ومستمر، وتكوين بيئة لغوية مواتية، باستخدام وسائل التعليم التي تسمح للطلاب بالاستماع و التفاعل مع الناطقين الأصليين، اعتياد الدافع بحيث تصبح اللغة الأجنبية سلوكًا معتادًا 12.

هناك العديد من أنشطة تعلم اللغة العربية التي يمكن تطوير ها بناءً على هذا المدخل، بما في ذللك :

${ }^{10}$ Iskndarwasid dan Hadang Sunendar, Strategi Pembelajaran Bahasa, (Bandung: PT Remaja Rosdakarya, 2011), hlm. 47

${ }^{11}$ Zalyana, Psikologi, hlm. 127

${ }^{12}$ Aziz Fachrurrazi dan Erta Mahyudin, Pembelajaran Bahasa asing,(Jakarta:Bania Publishing, 2010), hlm.38 
التعرف لمهار ات الاستماع والكلام كبداية في التعلم قبل تقديم مهار القراءة ومهارات

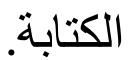

ب) ممارسة اللغة واستخدامها بشكل نشط ومستمر حتى يكتسب المتعلمون مهارات لغوية ويكونون في شكل عادة استخدام اللغة العربية.

ج) تكوسن بيئة لغوية التي تداعم لعملية تعويد التو اصل باللغة المتعلمة بشكل فعال. د) استخدام وسائل التعلم التي تسمح للمتعلمين بالاستماع والتفاعل مع الناطقين الأصليين (الناطق الأصلي). م) تشجيع المعلمين بأن يتحدثوا باللغة العربية الصحيحة ، ليكونو ا قدوة جيدة لطلابهم في اللغة

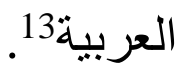

من العوامل التي تؤثر بشكل كبير وتحدد نجاح عملية تعلم اللغة العربية هي البيئة. وأهداف تكوين بيئة ال بالتو اصل باللغة العربية هي فيما يلي: أ) التعود على استخدام اللغة العربية اتصاليا، من خلال ممارسة المحادثة، والمناقثنة، و الندوات، و المحاضر ات والتعبير عن طريق الكتابة (التعبير التحريري). ب) تقديم التعزيز لاكتساب المو اد التي تم تعلمها في الفصل. ج) تنمية الإبداعية وأنشطة اللغة العربية التي تتكامل بين النظرية والتطبيق في جو غير رسمي مريح وممتع.

يتطلب تطبيق هذا المدخل من الطلاب إعادة التعبير عن المعرفة التي تعلموها في شكل تقارير أو اختبارات. يتبع عرض الموضوع ترتيب الأجزاء ككل. يؤكد التعلم والتقويم على هلى النتائج ، ويتطلب التقويم إجابات صحيحة. فالإجابة الصحيحة تبين أن الطالب قد أنهى در استه. يمكن للمدرسين تتظيم المواد التعليمية في شكل جاهز بحيث يتم نقل أهداف التعليم التي يجب أن يتقنها الطلاب بالكامل بواسطة المعلم. لا يلقي المعلم الكثبر من المحاضرات، ولكن

\footnotetext{
${ }^{13}$ Muhammad Mahmudi, "Penerapan Teori Behavioristik Dalam Pembelajaran BahasaArab(KajianTerhadap Pemikiran BF. Skinner)”, Prosiding Koferensi Bahasa Arab II (15 Oktober 2016), hlm. 433-434
} 
بإرشادات موجزة تتبعها أمثلة، إما بنفسه أو من خلال المحاكاة. يتم تتظيم المواد التعليمية في تسلسل هرمي من البسيط إلى الصعب. التعلم موجه نحو النتائج التي يمكن قياسها وملاحظتها. يجب تصحيح الأخطاء على الفور. يتم استخدام التكرار والتمارين بحيث يصبح السلوك مك

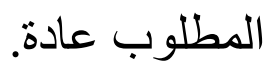

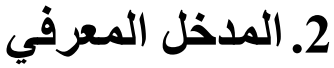

يؤكد مدخل التعلم المعرفي أن التعلم عملية تحدث في العقل البشري. في الأساس، التعلم هو عملية تجارية تتضمن أنشطة عقلية تحدث لدى البشر نتيجة لعملية التفاعل النشط مع بيئتهم للحصول على تغيير في شكل المعرفة والفهم والسلوك والمهار ات وقيم المواقف التي تكون نسبية ودائمة.

ففي التعلم، يؤكد المذهب المعرفي أهمية العوامل الداخلية للفرد في التعلم دون التساهل للعوامل الخارجية أو البيئية. ويعتقد هذا المدخل أن التعلم هو التفاعل بين الفرد و البيئة، وهو يحدث باستمرار طو ال حياته. المعرفة هي قطعة أثاث في أذهاننا وهو "المركز" الذي يقود الأنشطة المختلفة، ونحن نتعرف على البيئة، ونرى المشاكل المختلفة، ونحلل المشاكل المختلفة، ونبحث عن معلومات جديدة، و استخلاص النتائج وما إلى ذلك.

بالإضافة إلى ذللك، يعترف هذا المدخل بمفهوم أن التعلم هو نتيجة التفاعل المستمر بين الفرد و البيئة من خلال عملية الاستيعاب والتكيف. يكثف المدخل المعرفي أن التعلم الذي يقوم به الأفر اد هو نتيجة تفاعلاتهم العقلية مع البيئة المحيطة، مما يؤدي إلى تغييرات في المعرفة أو السلوك. في التعلم في هذا المدخل، يوصى باستخدام الوسائل الملموسة لأن الأطفال لا يمكنهم التفكير بشكل تجريدي حتى الآن.

في هذا المدخل، هناك مجالان للار اسة يهتمان بعملية التعلم أكثر من مخرجات التعلم ، 
أ) لا يقتصر التعلم على المثير والاستجابة فحسب بل يشمل أيضًا عملية تفكير معقدة للغاية14. ب) يُينى العلم في الإنسان من خلال عملية تفاعل مستمرة مع البيئة. ووفقًا لعلم النفس المعرفي، يُنظر إلى التعلم على أنه محاولة لفهم شيء ما من خلال ربط المعرفة الجديدة بهياكل التفكير الحالية. يتم تنفيذ هذا الجهد بنشاط من قبل الطلاب. يمكن أن يكون النشاط في شكل البحث عن الخبرة، والبحث عن المعلومات، وحل المشكلات، وملاحظة البيئة، وممارسة شيء ما لتحقيق هدف معين، بحيث تكون المعرفة التي كانت مملوكة من قبل ضرورية للغاية لنجاح تعلم معلومات جديدة.15. يفترض هذا المدخل أيضًا أن التعلم هو تتظيم الجوانب المعرفية و الإدر اكية لاكتساب الفهم. في هذا النموذج، يتم تحديد سلوك الثخص من خلال تصور اته وفهمه. وفي الوقت نفسه، يتم تحديد المواقف المتعلقة بالأهداف و التغيرات في السلوك إلى حد كبير من خلال عملية التفكير الداخلي التي تحدث أثناء عملية التعلم. من حيث المبدأ، التعلم هو تغيير في الإدراك و الفهم لا يمكن دائمًا اعتباره سلوكًا (لا يمكن ملاحظته دائمًا) 16. ويؤكد هذا المدخل على فكرة أن أجزاء الموقف التي تحدث في عملية التعلم مترابطة ككل. لذلك إذا نم تقسيم الوضع برمنه إلى مكونات صغيرة ودر استها بشكل منفصل، فهذا يعني

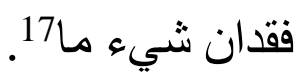

التدفق المعرفي له المعايير و الخصائص الرئيسية. فخصائص التدفق المعرفي التي يمكن

$$
\text { ب) بؤيتها هي كما يلي: }
$$

\footnotetext{
${ }^{14}$ Suyono dan Hariyanto, Belajar dan Pembelajaran; Teori dan Konsep Dasar, (Bandung: PT Rosda Karya, 2001), hlm. 75

${ }^{15}$ Muhaimin, Sutia'ah, Nur Ali, Paradigma Pendidikan Islam; Upaya Mengefektifkan PAI di Sekolah, (Bandung: PT Remaja Rosda Karya, 2002), hlm. 198

${ }^{16}$ Bambang Warsita, Teknologi Pembelajaran ; Landasan Dan Aplikasinya, (Jakarta: Rineka Cipta, 2008) hlm. 69.

${ }^{17}$ Muhaimin dkk, Paradigma Pendidikan, hlm. 199
} 
Tafhim Al-'Ilmi : Jurnal Pendidikan dan Pemikiran Islam Terakreditasi Nasional SK No : 148/M/KPT/2020
ISSN: 2252-4924, e-ISSN: 2579-7182

Volume 12, No. 1 September 2020

$$
\text { ج) يهيتم بالأدوار المعرفية. }
$$

و) يهتم بتكو ين بناء المعرفية.

بالنسبة لتطبيق مدخل التعلم المعرفي في تعليم اللغة، فيجب على المعلمين أن يفهموا أن الطلاب ليسوا من الكبار الساهلين في عملية تفكيرهم، يتعلم الأطفال في بداية سنهم المبكرة استخدام أثياء ملموسة، ونشاطهم تعد مهما للغاية ،يقوم المعلمون بتجميع المواد باستخدام نمط أو منطق معين من البسيط إلى المعقد، يقوم المعلم بإنشاء تعلم هادف، مع الإهتمام إلى الفروق الفردية للطلاب لتحقيق نجاح الطالب. بناءً على مبدأ مدخل معالجة المعلومات، تمت صياغة عدة مبادئ توجيهية لتطبيق مدخل معالجة المعلومات، وهي: أ) يجب أن يتأكد المعلمون من أن كل طالب لديه اهتمام بما يتعلمه. فلذلك، لأجل جذب انتباه الطلاب، يمكن للمدرس اتخاذ إجراء من خلال إعطاء إثارات معينة مثل التصفيق أو هات الدوس على السبورة، أو المشي في جميع أنحاء الغرفة أو التحدث على الإيقاع، وبدء الدرس بطرح أسئلة تثير اهتمام الطلاب بالموضوع الذي يتم تعليمه. ب) مساعدة الطلاب على التمييز بين المعلومات المهمة والمعلومات التي ليست مهمة لتركيز الانتباه، على سبيل المثال عن طريق كتابة أهداف التعليم، وشرح الوقت للتوقف والتكرار

$$
\text { مرة أخرى أو مطالبة الطلاب بتكر ار ما تم شرحه. }
$$

ج) مساعدة الطلاب على ربط المعلومات الجديدة بما هو معروف، على سبيل المثال عن

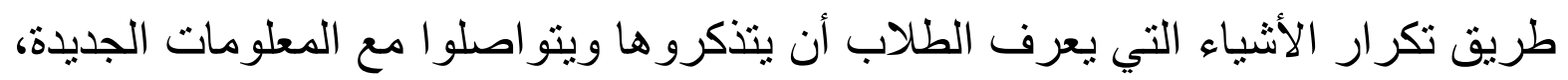
باستخدام المخططات أو الخطوط لإظهار علاقة المعلومات الجديدة بالمعلومات التي لديهر. د) اتاحة الفرصة لمراجعة المعلومات والتحقق منها عن طريق بدء الدروس بمراجعة الواجبات المنزلية، وإجر اء اختبار ات قصيرة متكررة، و إنثاء الألعاب أو طرح الطلاب لهاب وطرح الأسئلة على بعضهم البعض. 
o) تقديم الدروس بطريقة منظمة وو اضحة، على سبيل المثال شرح أهداف التعلم، وتقديم نظرة عامة أو ملخص. و) تفضيل التعلم الهادف، وليس الذاكرة، على سبيل المثال من خلال تعليم مفردات الكلمات الجديدة وربطها بكلمات مملوكة بالفعل.

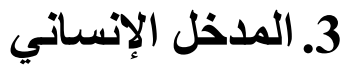

تأتي الإنسانية من أصل الكلمة اللاتينية "homo" و التي بمعنى الإنسان. فالإنسانية تعني أن تكون الإنسان إنسانيًا وفقًا لطبيعتهم. في البداية، كانت النزعة الإنسانية حركة تعزز كرامة الإنسان وقيمهم. كالمدرسة الفكرية النقدية نشأت الإنسانية من حركة تدعم درجة البشر، وتؤكد

$$
\text { الإنسانية على كر امة الإنسان و أدوار هم ومسؤولياتهم } 18 .
$$

في التعليم الإنساني، يتم تفسير التعلم على أنه عملية بشرية. لذلك يجب أن يحتوي التعليم الحديث على جوانب إنسانية للتعلم. لذللك، حان الوقت لتطوير التدريس والتعليم الإنساني في المؤسسات التعليمية في إندونيسياب1. يرى مدخل التعلم الإنساني البشر على أنهم أشخاص أحرار ومستقلون لتحديد اتجاه حياتهم. يتحمل البشر المسؤولية الكاملة عن حياتهم و أيضًا عن حياة الآخرين. المدخل الأكثر ملاءمة المستخدم في التعلم الإنساني هو المدخل الحواري، و المدخل التأملي، و المدخل التعبيري. دعا المدخل الحواري الطلاب إلى التفكير معًا بشكل نقدي وإبداعي. لا يعمل المربون كمعلمين ولكن كميسرين وشركاء في الحوار، يدعو المدخل التأملي الطلاب إلى الحوار مع أنفسهم، بينما يدعو المدخل التعبيري الطلاب إلى التعبير عن أنفسهم بكل إمكاناتهم. ولذلك لا يتولى المعلمون التوعية المسؤولية، بل يساعدون الطلاب وير افقونهم فقط في عملية التطوير الذاتي وتحديد المو اقف وفرز القيم التي سيتم النضال من أجلها2.

\footnotetext{
${ }^{18}$ Mangunhadjana, Isme-Isme dari A sampai Z. (Yogyakarta : Kanisius, 1997), hlm.93

${ }^{19}$ Moh. Amin Dkk. humanistic education, (Bandung: Departemen Penididikan dan Kebudayaan Dirjen Pendidikan Tinggi, 1979), hlm. 8

${ }^{20}$ Ende Supriyadi, Pendidikan dengan Pendekatan Humanistik, makalah, (Cianjur: t.p., 2011), hlm. 3
} 
يؤكد المدخل الإنساني على أن التعليم هو أولاً وقبل كل شيء كيفية إقامة الاتصالات و العلاقات الثخصية بين الأفر اد وبين الأفراد والجماعات في المجتمع المدرسي. هذه العلاقة تتطور بسر عة وتتنج ثمارًا تربويا إذا كانت قائمة على المحبة بينهما. يتطور الأفراد على النحو الأمثل نسبيًا دون عقبات عندما يكونون في جو من الحب (الحب غير المشروط) ، وقلب متفهم (فهم القلب) و علاقات شخصية فعالة (علاقات شخصية). في تعليم شخص ما، يجب أن نكون قادرين على قبول أنفسنا كما هم ثم التعبير عنها بصدق (عرض الأزياء). لا يقتصر التعليم على نقل المعرفة وتدريب المهارات اللفظية للطلاب فحسب، بل هو وسيلة مساعدة حتى يتمكن الطلاب من تطوير أنفسهم على النحو الأمثل 21. وفي مجلة التربية الإسلامية التربوي، قالت راتنا سيفا رشمهانا : إن مبادئ التعلم الإنساني وفقًا لكارل روجرز (Carl Rogers) تثمل الرغبة في التعليم، و التعلم الهادف، و التعلم بدون تهديدات، و التعلم بمبادرة شخصية، و التعلم من أجل التغيير22. التفسير المفاهيمي لكل من هذه المبادئ هو كما يلي: أ) أن تكون الرغبة في التعلم. فلكل البشر لديه رغبة طبيعية في التعلم. يتضح هذا من خلال فضول الأطفال الثديد عندما تتاح لهم الفرصة لاستكثناف البيئة. هذا الدافع للفضول للتعلم هو افتراض أساسي للتعليم الإنساني. في الفصل الدراسي الإنساني، يتم منح الأطفال الفرصة والحرية لإرضاء فضولهم، وتحقيق اهتماماتهم واكتشاف ما هو مهم وذو مغزى عن البيئة حولهم. ب) أن يكون التعلم ذات معنى. فسيكون التعلم ذات معنى إذا كان ما تم تعلمه وثيق الصلة باحتياجات ونوايا الطلاب. هذا يعني أن الطلاب سيتعلمون بسر عة إذا كان ما تم تعلمه له

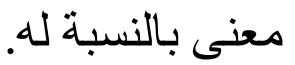

\footnotetext{
${ }^{21}$ Endi Supriyadi, Pendidikan Dengan, hlm. 3

${ }^{22}$ Endi Supriyadi, Pendidikan Dengan, hlm. 3
} 
ج) التعلم بدون تهـيد/ت. التعلم سهل ويمكن تخزين النتائج بشكل جيد في بيئة خالية من التهديدات. ستعمل عملية التعلم بسلاسة عندما يتمكن للطلاب من اختبار قدر اتهم، ويمكنه التجربة بتجربة جديدة أو ارتكاب أخطاء بدون انتقاد الذي عادة ما يسيء إليهر.

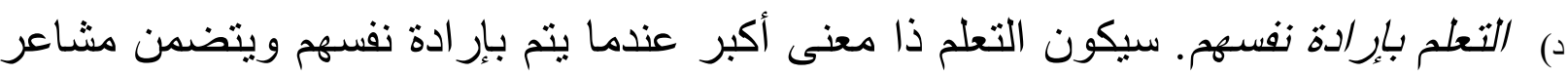
و أفكار المتعلم. إن القدرة على اختيار اتجاه التعلم الخاص بهم أمر ؤثر للغاية ويمنح الطلاب الفرصة لتعلم كيفية التعلم. يركز التعلم من تلقاء نفسه انتباه الطلاب على كل من العملية ونتائج التعلم. بالإضافة إلى ذلك، فإن تعليم الطلاب أن يكونوا أحرارًا ومستقلين وو اثقين من أنفسهر. ه) أن بكون التعلم من أجل التغبير. التعلم الأكثر فائدة هو التعرف على عملية التعلم. وفقًا لروجرز (Rogers)، أن الطلاب تعلموا في الماضي عن الحقائق والأفكار الثابتة، وكان العالم حينذاك بطينًا في التغيير، وكان ما يتم الحصول عليه في المدرسة يعتبر كافياً لتلبية احتباجات العصر. ولكن في الوقت الحاضر التغيير هو حقيقة أساسية في الحياة، و العلوم و التكنولوجيا تتقدم وتتقلم دائمًا. وبالتالي، فإن المطلوب الآن هو أن يتمكن الناس من التعلم في البيئة المتغيرة وستستمر في التغيير. Frank G. في غضون ذلك، أعرب آرثر كومبس (Arthur Combs) في فر انك ج.جوبل (Goble

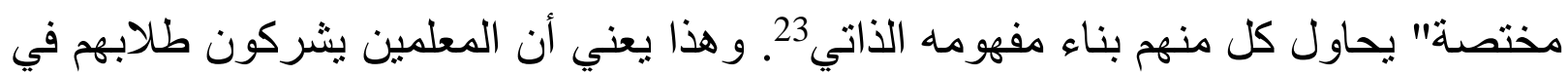
عملية التعليم، بحيث يكون لديهم تجارب ناجحة، ويشعرون بالقبول والاحترام والإعجاب

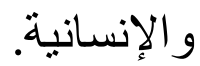

\footnotetext{
${ }^{23}$ Frank Goble, Madzhab Ketiga-Psikologi Humanistik Abraham Maslow, (Yogyakarta: Kanisius, 1987), hlm. 120
} 
في عملية التعلم في الفصل الدراسي، ذكر محمد أمين وآخرون في كتابه "التعليم الإنساني" أنه يمكن تحقيق علم النفس الإنساني بعدة مداخل24 ، وهي:

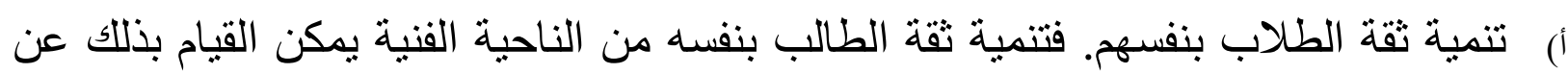
طريق طرح أسئلة منل، "في عملية التدريس حتى الآن، ما هي المهمة التي تعنقد أنها الأكثر إرضاءٌ؟"، بمثل أسئلة من هذا القبيل، يرجى أن يتم تكوين التصور للنجاح مما سيزيد من الثقة بالنفس لدى الطلاب. ب) تتمية الإبداعية، من خلال تنمية الكفاءة الإبداعية لدى الطلاب، لأن الإنسان يمتلك إمكانات إبداعية. ويختلف الإبداع بين الإنسان و الحيوان، و عندما نقوم بأنشطة، فإن مفهومنا الذاتي ينمو بحيث يصبح أقوى كأفراد. والأسلوب المقترح لجعل الفصل مبدعًا هو العصف إنف الذهني، وهو تقديم مشكلة ويُطلب من الطلاب تقديم أفكار هم، ثم يُطلب منهم مراجعة الأفكار بحيث بمكن استخدام النتائج لحل المشكلة. ج) توضيح القيم وتتمية الأخلاق. تهدف هذا المدخل إلى مساعدة الطلاب في نطوير العمليات المستخدمة في تحديد القيم الخاصة بهر. من الناحية الفنية ، يقدم المعلم المشكلات التي يمكن أن تشجع الطلاب على تحديد قيمهم الخاصة أو حل المشكلات التي تحتوي على نوعين من القيم المتضاربة. د) مدخل تعدد المواهب. من أجل تتمية المواهب بجانب الكفاءات الأكاديمية. يمكن القيام بذلك عن طريق تقديم عرض للطلاب، "من يستطع أن يكتب القصة تحت موضوع "الو الدين"؟". هذا السؤال لمعرفة هناك من الطلاب الذين لديهم موهبة في مجال الاتصـال.

${ }^{24}$ Moh. Amin, Dkk. humanistic education, hlm. 


\section{تعليم اللغة العربية المتأسس على المدخل الإنساني}

يركز تعلم اللغة العربية القائم على الإنسانية بشكل أكبر على كيفية تعلم اللغة ممتعة وليست إلحاحية. يكون تعلم اللغة العربية القائم على الإنسانية كما يلي: - تعليم اللغة المفتوح. تعليم اللغة المفتوح هو عملية تعليمية تعطى للطلاب الفرصة للتنقل بحرية في جميع أنحاء الفصل واختيار أنشطة التعلم الخاصة بهر، ويعمل المعلم فقط كدليل. وفي عملية التعلم المفتوحة هذه، تختلف البيئة المادية للفصل الدراسي عن الفصول الدراسية التقليدية. لأن الطلاب يعملون بشكل فردي أو في مجموعات صغيرة. في هذه العملية، يتطلب الأمر وجود مراكز تعليمية أو مراكز نشاط في الفصل الدراسي تتيح للطلاب استكثاف مهار ات اللغة العربية. الموضوعات ذات الصلة باللغة العربية. مهارات أو اهتمامات محددة للطلاب مرتبطة بتعلم اللغة العربية. يمكن أن يوفر هذا المركز تعليمات لدر اسة موضوع في غياب المعلم ويمكنه تسجيل مشاركة الطلاب وتقدمهم لمناقشتها لاحقًا

$$
\text { مع المعلم } 25 .
$$

- تعلم اللغة التعاونسي. يعد تعلم اللغة التعاوني أساسًا جيدًا لزيادة الدافع لنجاح الطلاب. في

$$
\text { التمرين. التعلم التعاوني له ثلاث خصائص ؛ }
$$

يعمل الطلاب في فرق تعليمية صغيرة (4-6 أعضاء). ويبقى هذا التكوين لمدة أسبوع. 2) يتم تشجيع الطلاب على مساعدة بعضهم البعض في در اسة المو اد الأكاديمية والقيام بذلك في مجمو عات ؛ و3) يتم منح المتعلمين مكافآت أو جو ائز بناءً على إنجاز المجمو عة26. هناك 4 (أربعة) أنواع من التقنيات في التعلم التعاوني. وهي؛ أ) بطولة ألعاب الفريق(Team-Games-turnament). في هذه التقنية، يتحد الطلاب ذوو القدرات و الأجناس المختلفة في فريق يتكون من أربعة إلى خمسة أعضاء. بعد أن يقدم المعلم مادة الدرس. ثم عمل الفريق على أوراق العمل. نسأل بعضنا البعض أسئلة. ويدرس معًا

\footnotetext{
${ }^{25}$ Rumini. S. dkk. Psikologi Pendidikan, ( Yogyakarta: Fakultas Ilmu Pendidikan Universitas NegeriYogyakarta, 1993), hlm. 111

${ }^{26}$ Ibid, hlm. 110
} 
للاستعداد لسباق أو بطولة مرة واحدة في الأسبوع. في البطولة، يتم تحديد أعضاء الفريق بناءً على القدرة في الأسبوع السابق. النتائج. يتمتع الطلاب الذين حققوا أقل إنجاز في كل مجموعة بنفس الفرصة لكسب النقاط لفريقهم مثل الطلاب الذين حققو أعلى إنجاز. أما بالنسبة لمسار البطولة، فقد تناوب الطلاب على أخذ البطاقات والإجابة على الأسئلة المدرجة على البطاقة. أي الأسئلة التي تتطابق مع المادة التي تم دراستها خلال ذلك الأسبوع في نهاية البطولة. يعد المعلم الورقة التالية حول الفرق التي نجحت وأعلى الدرجات التي تم تحقيقها. على الرغم من بقاء عضوية الفريق كما هي. ومع ذلك، قد يتغير الأشخاص الثناثة الذين يمثلون الفريق للمنافسة بناءً على مظهر و إنجاز ات كل عضو. على لـ سبيل المثال، تحصيل الطالب حاليًا منخفض ويتتافس مع طلاب آخرين بقدرات مماثلة. ثم في الأسبوع التالي كان يمكن أن ينافس الطلاب المتفوقين عندما يتحسن ؛ ب) مجموعة الطلاب لشعب الإنجاز (Student teams-Achivement Divisions). تستخدم هذه التقنية مجموعة من أربعة إلى خمسة أعضاء. ومع ذلك ، تم استبدال نشاط البطولة بسؤال بعضنا البعض لمدة خمس عشرة دقيقة. حيث تم ترتيب الأسئلة التي نم طرحها أولاً بواسطة الفريق. يتم تحويل درجات الأسئلة إلى درجات الفريق. حصلت أعلى الدرجات على نقاط أكثر من الدرجات الأقل. بالإضافة إلى أن هنالك أيضًا درجة تحسن ؛ إنى ج) بانور اما. يتم وضع الطلاب في فرق صغيرة غير متجانسة. ثم حصل الفريق على مواد لتعلم اللغة العربية. يتعلم الطلاب الأقسام الخاصة بهم مع أعضاء الفريق الآخرين الذين

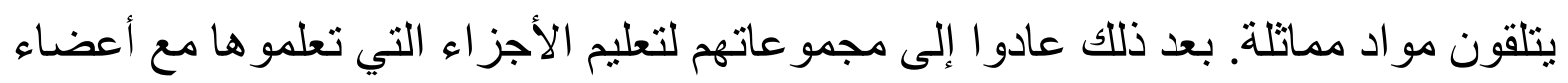

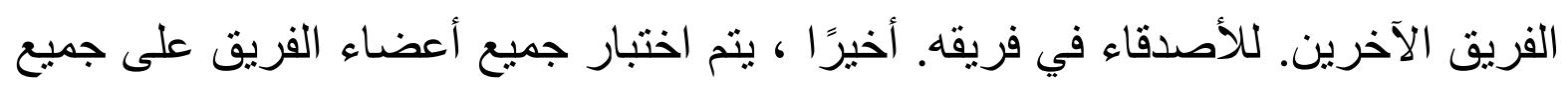
المواد التعليمية. يمكن تحديد الدرجات التي حصل عليها الطلاب بطريقتين. وهي النتيجة لكل طالب و النتيجة المستخدمة لإنشاء نتيجة الفريق ؛ د) التحقيق الجماعي (Group Investigation). يعمل الطلاب هنا في مجموعات صغيرة للرد على مشاريع الفصل المختلفة المتعلقة بتعلم اللغة العربية. تقسم كل مجموعة المهمة إلى لى 
مواضيع فرعية يتم تعيينها لكل عضو في المجموعة للبحث فيها من أجل تحقيق أهداف المجمو عة. بعد ذللك قدمت كل مجمو عة نتائج بحثنها إلى الفصل 27.

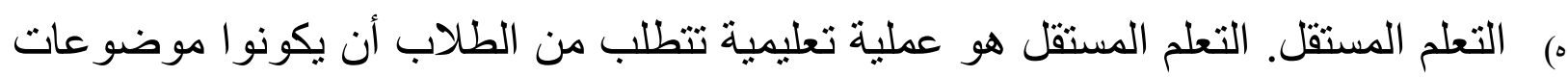
عليهم تصميمها. تنظيم ومراقبة أنشطتهم الخاصة بطريقة مسؤولة. يتطلب تنفيذ التعلم المستقل استقلالية كبيرة عن الطلاب، وهنا يصبح المربي ميسر اً. وأن تصبح مكانًا لطرح الأسئلة وحتى من المؤمل أن يكون التعليم خبيرًا في المجال الذي يتعلمه الطلاب.

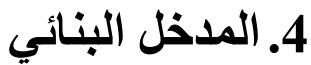

البنائية هي فلسفة المعارف التي تعتقد بأن المعرفة هي نتيجة تكوين بناء المعرفة من

$$
\text { المتعلم. هذا يعني أن كل شخص بشكل معرفته الخاصة.28. }
$$

المعجم الدولي للتربية عرف البنائية بأنها : "رؤية في نظرية التعلم ونمو الطفل قوامها أن الطفل يكون نشطا في بناء أنماط التفكير لديه نتيجة تفاعل قدر اته الفطرية دع الخبرة". وتعرفها جامعة مدرسة التربية بجامعة كولور ادو بأنها : "فلسفة التعلم القائمة على الإفتر اض القائل بأننا نبني فهنا للعالم الذي نعيش فيه بالإعتماد على خبر اتنا"29. يؤكد هذا الفهم أن الجميع في الواقع بنّاءون. المعرفة ليست "شينًا موجودًا بالفعل" وتأخذها فقط، ولكنها تكوين مستمر للشخص الذي يتعلم مع إعادة التنظيم في كل مرة بسبب فهم جديد. النظرية البنائية لها جذور تاريخية تبلغ ذروتها في Piaget و Vygotsky ، وهما عالمان شددا على أن التغيير المعرفي يحدث حتمًا عندما تمر المعارف القديمة في عملية عدم التوازن social ( بالمعلومات الجديدة. بالإضافة إلى ذلك، أكد كلاهما أيضًا على أهمية التعلم الاجتماعي

\footnotetext{
${ }^{27}$ Ratna Syifa'a Rachmahana "Psikologi Humanistik dan Aplikasinya dalam Pendidikan". ElTarbawi: Jurnal Pendidikan Islam (2012), hlm. 10-11 http://journal.uii.ac.id/.

${ }^{28}$ Slamet Suyanto, Dasar-dasar Pendidikan Anak Usia Dini, (Yogyakarta: Hikayat Publishing, 2005)hal. 147

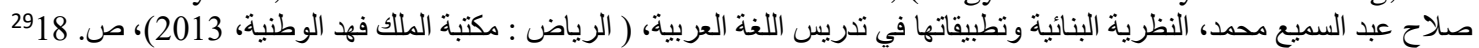


(nature learning المفاهيمي و أكد فون غلاسبرفيلد أن المعرفة ليست تقليدًا للواقع بالنسبة للبناء، المعرفة هي بناء العقل البشري. المعرفة هي إطار لفهم كيفية تنظيم الناس للخبرات وما بعتقدون أنه حقائق 31.

تفترض البنائية أن المعرفة هي نتيجة البناء البشري. يبني البشر معرفتهم من خلال تفاعلهم مع الأشياء و الظواهر والتجارب وبيئته. بالنسبة للبناء، لا يمكن نقل المعرفة ببساطة من شخص إلى آخر، ولكن بجب أن يفسر ها كل شخص. المعرفة لبست شبيًًا تم صنعه، ولكنها عملية تتطور باستمر ار 32. يمكن تقسيم البنائية إلى ثلاثة أنواع بالإعتماد على من أو أ شيئ الذي بعين تكوين المعرفة. أولاً، البنائية النفسية الثخصية ، التي تؤكد أن الثخص هو من يبني المعرفة. ثانيًا ، البنائية الاجتماعية، التي تؤكد على أن المجتمع هم الذين يبنون المعرفة. ثالثًا، الثقافة الاجتماعية التي تستخدم كلاً من البناء الثخصي والاجتماعي، حيث يرتبط الجانبان في تكوين المعرفة. فيما يلي خصائص التعلم القائم على البنائية وفقًا لـ ( Driver and Oldham 1994 : أ) التوجيه، حيث يتم منح الطلاب الفرصة لتطوير الدافع في تعلم موضوع ما وتوفير الفرص

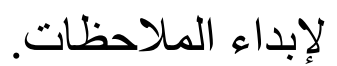
ب) الاستتاج، أي التعبير عن الفكرة من خلال المناقشة عن الكتابة وعمل الملصقات ونحو ذلك. ج) إعادة تنظيم الفكرة، أي تصنيف الأفكار مع أفكار الآخرين من خلال تصنيف أفكار جديدة ، وتقييم الأفكار الجديدة.

30 https/benrampt.wordpress.com/ pendekatan- konstruktivistik- dalam- pembelajaran- resume chapter- 8- buku Eduacational Psychology karya Robert E Slavin, akses tanggal 10 September 2019.

${ }^{31}$ Paul Suparno, Filsafat Konstruktivisme Dalam Pendidikan, (Yogyakarta: Kanisius, 2001), hlm. 18.

${ }^{32}$ Ibid. hlm. 28-29. 
ه) تطبيق استخدام الأفكار الجديدة في المواقف المختلفة، أب الأفكار أو المعرفة التي تم تشكيلها في مو اقف مختلفة. و) مراجعة، أي تطبيق المعرفة، الأفكار الحالية تحتاج إلى المراجعة عن طريق الإضافة أو

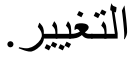
يقول Von Glaserfed (في Paul، 1996) ، أن هناك حاجة إلى العديد من القدر ات في عملية

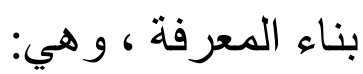
أ) القدرة على تذكر التجربة واسترجاعه. ب) القدرة على المقارنة و اتخاذ القرار ات بشأن أوجه التشابه والاختلاف حول الثيء. ه) القدرة على تفضيل تجربة ما على أخرى (الضمير الانتقائي).

العو امل التي تحد من عملية بناء المعرفة

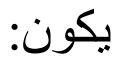
أ) نتائج البناء التي يمتلكها شخص ما (معرفة مبنية). ب) مجال خبرة الشخص (domain of experience). ج) شبكة البنية المعرفية للشخص (existing cognitive structure). دور المعلم في المدخل البنائي والذي يتضمن أنشطة كوسيطو ويسر للطلاب: أ) تقديم خبر ات تعليمية لمحاسبة الطلاب. ب) تقديم أو توفير الأنشطة التي تشجع حماسة الطلاب وتساعدهم على التعبير عن أفكار هم. ج) إقامة الملاحظة و التقويم لمعرة مدى تفكير الطلاب يجري أم لا. بعض الأثياء المهمة حول التقييم في التدفق البنائي هي: أ) موجهة نحو الو اجبات الحقيقية ؛ ب) بناء المعرفة التي تصف عملية التفكير العليا ج) بناء الخبر ات الطلابية. 
د) توجيه التقويم في سياق واسع مع وجهات نظر متعددة. فيما يلي وصف لخصائص التعلم البنائي وفق العديد من المر اجع وهي: أ) المعرفة مبنية على الخبرة أو المعرفة الموجودة مسبقًا. ب) التعلم هو تفسير شخصي للبيئة. ت) التعلم هو عملية نشطة يتم فيها تطوير المعنى بناءً على التجربة. ث) تنمو المعرفة بسبب التفاوض (negosiasi) على المعنى من خلال المعلومات المختلفة أو الاتفاق على وجهة نظر في التفاعل أو التعاون مع الآخرين.

ج) يجب أن يقع التعلم في بيئة واقعية، ويجب أن يتكامل التقييم مع المهمة وليس مع نشاط منفصل33.

الأساليب التي يتبعها المعلمون في مدخل النظرية البنائية في جوهر ها، هي التعلم النشاط يتم تنفيذه لتكوين الجو أو تقديم الخدمات بحيث يتعلم الطلاب. وفي تكوين الجو أو تقديم الخدمة، فإن الثيء الأساسي هو أن يفهم المعلمون كيفية حصول طلابهم على المعرفة من أنشطة التعلم الخاصة بهم. إذا تمكن المعلم من فهم عملية اكتساب المعرفة، فيمكنه عندئذ تحديد استر اتيجية أو أساليب التعلم المناسبة لطلابه. من الصعب بالفعل معرفة حدوث عملية التعلم لدى الطلاب الذين يتعلمون بالعين المجردة، لأن عملية التعلم تتم عقليًا. ودع ذلك، من خلال النتائج المختلفة للأبحاث أو التجارب، يمكن لعلماء النفس وصف كيفية حدوث هذه العملية. يجادل علماء النفس البنائيون بأن عملية اكتساب المعرفة تتم من خلال إعادة هيكلة الهياكل المعرفية الحالية لتتناسب مع المعرفة التي سيتم الحصول عليها بحيث يمكن تكييف المعرفة. في عملية التذريس والتعلم، هناك حاجة إلى طريقة أو طريقة لتحقيق أهداف التعلم. 
ووفقًا ل Oemar Hamalik، فإن طريقة التدريس هي طريقة أو تقنية أو خطوات سيتم اتخاذها في عملية التعليم والتعلم33. وفي الوقت نفسه، Roestiyah تؤكد أن طريقة التدريس هي تقنية عرض يتقنها المعلم لتدريس أو تقديم المواد التعليمية للطلاب في الفصل بحيث يمكن للطلاب التقاط الدرس وفهمه و استخدامه بشكل صحيح 35. هناك طرق مختلفة يمكن للددرسين استخدامها في أنشطة التعلم، بما في ذلك ؛ طريقة المحاضر ات المتنو عة، طريقة السؤال و الجو اب، طريقة المناقثنة، طريقة التكليف، طريقة لعب الدور، طريقة الرحلة الميدانية، طريقة الاستفسار، طريقة العمل الجماعي، طريقة الاكتشاف. لا يمكن استخدام جميع الأساليب نظرًا لمحدودية الإمكانيات و الوقت. ومع ذلك، فإن الثيء الأكثر أهمية هو أن استخدام الطريقة يجب أن يكون مرتبطًا بالوضع وأهداف التعلم المراد تحقيقها و التأكيد على نثاط الطلاب في بناء المعرفة.

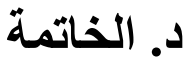

من المناقنشة على النحو الوارد أعلاه عن المداخل النفسية وتطبيقاتها في تعليم اللغة العربية يمكن استخلاص نقاط الاستتناج التالية: 1. أن هناك أربعة مداخل التعليمية تندرج في فئة المداخل النفسية ، وهي: المدخل السلوكي ، و المدخل المعرفي ، و المدخل الإنساني ، و المدخل البنائي. 2. المدخل السلوكي هو المدخل الذي يؤمن بأن ذكاء الثخص أو معرفته تتشكل بناءً على ،لى نتائج تفاعله مع البيئة. يؤكد هذا المدخل على دور البيئة ويؤكد على تكوين ردود الفعل أو الاستجابات. بالإضافة إلى ذلك، يؤكد هذا المدخل أيضًا على أهمية التدريب، و الميكانية لنتائج التعلم، ويؤكد على دور القدرة. نتيجة التعلم التي تم الحصول عليها وهي ظهور

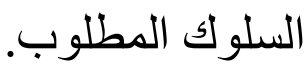

${ }^{34}$ Oemar Hamalik, Proses Belajar Mengajar, ( Jakarta : Bumi Aksara, 2003), hlm. 2

${ }^{35}$ Roestiyah NK. Strategi Belajar Mengajar, (Jakarta : Rineka Cipta, 2001), hlm. 1 
3. مدخل التعليم المعرفي هو المدخل الذي يعتقد أن التعلم هو عملية تحدث في العقل البشري. وفقًا لهذا المدخل، فإن التعلم هو عملية تتضمن نشاطًا عقليًا يحدث لدى البشر نتيجة لعملية تفاعل نشطة مع بيئتهم للحصول على تغيير في شكل المعرفة والفهم والسلوك و المهار ات و قيم المو اقف النسبية و الدائمة. 4. مدخل التعليم الإنساني هو المدخل الذي يرى البشر كموضوعات مستقلة في تحديد اتجاه حياتهم. إنه مسؤول مسؤولية كاملة عن حياته وكذلك عن حياة الآخرين. يتم تطبيق هذا المدخل في التعلم بعدة طرق و هي طريقة الحوار، وطريقة التأمل، و طريقة التعبير. يدعو طريقة الحوار الطلاب إلى التفكير معًا بشكل نقدي وإبداعي. لا يعمل المربون كمعمين ولكن كميسرين وشركاء في الحوار؛ يدعو طريقة التأمل الطلاب إلى الحوار مع أنفسهم، بينما يدعو طريقة التعبير الطلاب للتعبير عن أنفسهم بكل إمكاناتهم (تحقيق الذات). وبالتالي، لا يتولى اختصاصيو التوعية المسؤولية، بل بيساعدون الطلاب وير افقونهم فقط في عملية التطوير الذاتي، وتحديد المواقف وفرز القيم التي سيتم النضال من أجلها. 5. المدخل البنائية هو المدخل الذي يعتقد أن المعرفة هي نتيجة البناء البشري. يبني البشر معرفتهم من خلال تفاعلهم مع الأشياء والظواهر والتجارب وبيئتهر. يمكن تطبيق هذا المدخل في تعلم اللغة العربية بعدة طرق، بما في ذلك طريقة المحاضر ات المختلفة، طريقة السؤال والجواب، طربقة المناقنتة، طريقة التخصيص، طربقة لعب الأدوار، طريقة الرحلات الميدانية، الاستفسا، طريقة العمل الجماعي، طريقة الاكتشاف، طريقة العروض التوضيحية. عبد المجيد عيساني. نظريات التعلم وتطبيقها في علوم اللغة. (القاهرة : دار الكتب الحديث. 


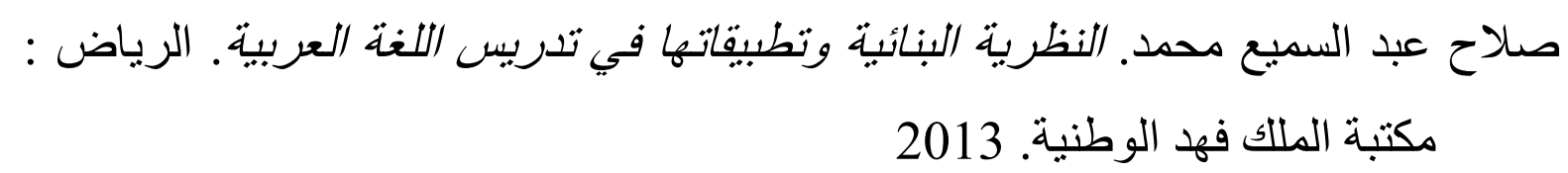

Abdul Wahab Rosyidi \& Umi Mahmudah. Aktive Lerning Dalam Pembelajaran Bahasa Arab. Malang : UIN-MALANG PRESS. 2008

Amin, Moh. Dkk. humanistic education. Bandung: Departemen Penididikan dan Kebudayaan Dirjen Pendidikan Tinggi. 1979.

Aziz Fachrurrazi dan Erta Mahyudin. Pembelajaran Bahasa asing. Jakarta:Bania Publishing. 2010.

Efendy, Ahmad Fuad. Metodologi Pengajaran Bahasa. Malang : Misykat. 2012.

Goble, Frank. Madzhab Ketiga -Psikologi Humanistik Abraham Maslow. Yogyakarta: Kanisius. 1987.

Hamalik, Oemar. Proses Belajar Mengajar. Jakarta : Bumi Aksara. 2003.

https/benrampt.wordpress.com/ pendekatan- konstruktivistik- dalam- pembelajaran- resume chapter- 8- buku Eduacational Psychology karya Robert E Slavin, akses tanggal 10 September 2019.

Iskndarwasid dan Hadang Sunendar. Strategi Pembelajaran Bahasa. Bandung: PT Remaja Rosdakarya. 2011.

Mahmudi, Muhammad. "Penerapan Teori Behavioristik Dalam Pembelajaran Bahasa Arab (Kajian Terhadap Pemikiran BF. Skinner)". Prosiding Koferensi Bahasa Arab II (15 Oktober 2016.

Mangunhadjana. Isme-Isme dari A sampai Z. Yogyakarta : Kanisius. 1997.

Muhaimin, Sutia'ah, Nur Ali. Paradigma Pendidikan Islam; Upaya Mengefektifkan PAI di Sekolah. Bandung: PT Remaja Rosda Karya. 2002.

NK,Roestiyah. Strategi Belajar Mengajar. Jakarta : Rineka Cipta. 2001.

Novan, Ardy Wiyani. Desain Pembelajaran Pendidikan: Tata Rancang Pembelajaran Menuju Pencapaian Kompetensi. Yogyakarta: Ar-Ruzz Media. 2013.

Pranowo. Teori Belajar Bahasa.Yogyakarta : Pustaka Pelajar. 2014.

Rachmahana, Ratna Syifa'a. "Psikologi Humanistik dan Aplikasinya dalam Pendidikan". ElTarbawi: Jurnal Pendidikan Islam (2012), http://journal.uii.ac.id/.

Rumini. S. dkk. Psikologi Pendidikan. Yogyakarta: Fakultas Ilmu Pendidikan Universitas NegeriYogyakarta. 1993. 
Tafhim Al-'Ilmi : Jurnal Pendidikan dan Pemikiran Islam Terakreditasi Nasional SK No : 148/M/KPT/2020
ISSN: 2252-4924, e-ISSN: 2579-7182

Volume 12, No. 1 September 2020

Suparno,Paul. Filsafat Konstruktivisme Dalam Pendidikan, Yogyakarta: Kanisius, 2001

Supriyadi, Ende. Pendidikan dengan Pendekatan Humanistik. Makalah. Cianjur: t.p., 2011

Suyanto, Slamet. Dasar-dasar Pendidikan Anak Usia Dini, Yogyakarta: Hikayat Publishing, 2005

Suyono \& Hariyanto, Belajar dan Pembelajaran. Bandung : Remaja Rosdakarya. 2014.

Suyono dan Hariyanto. Belajar dan Pembelajaran; Teori dan Konsep Dasar. Bandung: PT Rosda Karya. 2001.

Warsita, Bambang. Teknologi Pembelajaran ; Landasan Dan Aplikasinya. Jakarta: Rineka Cipta. 2008.

Yuleilawati, E. Kurikulum dan Pembelajaran; Filosofi, Teori, dan Aplikas. Jakarta : Pakar Raya. 2004.

Zalyana. Psikologi Pembelajaran Bahasa Arab. Pekanbaru: Almujtahadah Press. 2010. 